\section{Outcomes following 25-gauge vitrectomies}

\begin{abstract}
Purpose Twenty-five gauge vitrectomy surgery offers potential advantages over standard 20-gauge vitrectomy surgery, but the short- and long-term post-operative complications, such as cataract formation, are still being evaluated. This study quantifies the outcomes seen following 25-gauge vitrectomies.

Methods This is a retrospective, consecutive, non-comparative case series of 25-gauge vitrectomies performed between January 2002 and August 2004. Cases without at least 3 months of follow-up and previous vitrectomies were excluded. Analyses were performed with $t$-test and Kaplan-Meier curves.

Results Seventy-one cases met inclusion criteria. The mean age of the patients was 65 years old (SD 11 years). A variety of surgical indications were included. A statistically significant difference was seen between the mean preoperative visual acuity (20/100) and the mean visual acuity at the 3-month post-operative visit $(20 / 60 ; P<0.0001)$.
\end{abstract}

${ }^{1}$ Department of Ophthalmology, Mayo Clinic, Rochester, MN, USA

${ }^{2}$ Retina Consultants Inc., Charleston, WV, USA

Correspondence:

CA McCannel, Department of Ophthalmology, Mayo Clinic, 200 First Street SW, Rochester, MN 55905, USA Tel: + 1507284 4567; Fax: + 15072844612. E-mail: mccannel.colin@ mayo.edu

Received: 14 November 2006

Accepted in revised form: 7 March 2007

Published online: 20 April 2007

None of the authors hold any proprietary interests. To the best of our knowledge and research of articles in English on PubMed and Ovid, there is no previous publication in print that displays what has been presented in this paper
A Kaplan-Meier curve established that for all cases $63.4 \%$ of eyes required cataract extraction at 1 year. Total mean follow-up time was $8.6 \pm 5.5$ months.

Conclusions Statistically significant improvement was seen in mean vision by 3 months following 25-gauge vitrectomy. Cataract formation after 25-gauge vitrectomies remains an important consideration. Eye (2008) 22, 1024-1028; doi:10.1038/sj.eye.6702819; published online 20 April 2007

Keywords: vitrectomy; cataract; 25-guage;

20-guage; complication; hypotony

\section{Introduction}

The potential advantages offered by sutureless, 25 -gauge vitrectomy surgery over the 20-gauge
LJ Faia'1, CA McCannel', JS Pulido'1, RM Hatfield², ME Hatfield ${ }^{2}$ and VEW McNulty ${ }^{2}$

vitrectomy surgery include less manipulation of the globe, decreased surgical time, decreased recovery time to preoperative visual acuity (VA) and reduced need for sutures. Previous reports have established the short-term safety and efficacy of 25-gauge vitrectomy in a variety of vitreoretinal disorders. ${ }^{1-4}$ Potential risks, including retinal or vitreal incarceration, hypotony, endophthalmitis, and retinal traction, have been reported, but the rate of post-operative cataract extraction with 25-gauge vitrectomy surgery has not been quantified previously by a Kaplan-Meier curve analysis. $^{5-7}$

We report visual outcomes, post-operative intraocular pressures, and cataract extraction rates for a series of 25-gauge vitrectomy surgeries.

\section{Methods}

The study consists of a retrospective, consecutive, non-comparative case series. All cases of 25-gauge vitrectomy surgeries between January 2002 and August 2004 by two vitreoretinal surgeons (CAM and $\mathrm{RMH}$ ) were reviewed. Cases with less than 3 months of follow-up and previous vitrectomies were excluded from analysis.

This study was approved by the Institutional Review Board and followed all federal, state and local laws regarding clinical research. All research followed the Tenets of the Declaration of Helsinki as well. The data collected included age, gender, pre-operative diagnosis, surgical indication, pre-operative Snellen VA, presence or absence of macular oedema, lens status, air-fluid exchange and/or gas-air exchange status and gas used. Additional post-operative data included intraocular pressure (IOP) on post-operative day (POD) 1, best recorded Snellen VA at 1 week, 6 weeks and 3 months and last follow-up, lens status at 1 week, 6 weeks, and 3 months post-operatively and last follow-up. 
Earlier to surgery, informed consent was obtained. A standard, 25-gauge three-port pars plana vitrectomy under monitored anaesthesia care with regional retrobulbar block was performed. Either the Alcon ACCURUS (Fort Worth, TX, USA) or Bausch and Lomb Millennium (San Dimas, CA, USA) 25-gauge system was used. All cases used the binocular indirect ophthalmomicroscope (BIOM) system and a fundus contact lens for macular work as necessary. At the conclusion of the surgery, before air-fluid exchange, the periphery was inspected with scleral depression for breaks, and if present, treated with endolaser or cryotherapy. Air-fluid exchange with gas mixture or air exchange was performed at the surgeon's discretion. Gases left in the eye included air, sulphur hexafluoride mixture, or perfluoropropane mixture.

For statistical analyses, visual acuities were converted to $\log$ MAR values. A paired, two-tiered Student's $t$-test was used to evaluate comparisons between preoperative and post-operative visual acuities. An unpaired student $t$-test was used for statistical analysis of IOP on POD 1 for all cases. An unpaired $t$-test comparing the POD 1 IOPs of eyes injected with gas to those without a gas injection was also carried out.

The cataract extraction rate was estimated using the Kaplan-Meier method. One curve established the cataract extraction rate for all cases. Subgroup analyses were then performed by dividing the eyes into two groups, eyes with gas injection and eyes without gas injection, and Kaplan-Meier curves were created to establish cataract extraction rates in these subgroups. Log-rank tests were used to compare the Kaplan-Meier curves for these subgroups.

\section{Results}

Seventy-one cases with at least 3 months of follow-up were identified and analyzed. There were 39 women and 32 men with a mean age of 65 (SD 11) years. Eight patients were pseudophakic before surgery. The other 63 patients were phakic. Preoperative surgical indications were epiretinal membrane (ERM) 39 eyes (55.0\%), macular hole (MH) 22 eyes (31.0\%), vitreous haemorrhage $(\mathrm{VH})$ six eyes $(8.4 \%)$, dense vitreous opacity one eye $(1.4 \%)$, and vitreomacular traction syndrome (VMT) three eyes (4.2\%). Additional secondary diagnoses included vitreomacular traction syndrome (4), diabetic macular oedema (4), and proliferative diabetic retinopathy (6). The total mean follow-up time was 8.6 months with a SD of 5.5 months (3.1-14.1 months). Overall, a statistically significant difference was seen between the mean preoperative VA (20/100) and the 3-month mean post-operative VA $(20 / 60)$ for all cases $(P<0.0001)$. The mean time required to return to preoperative VA was 5.6 weeks with a SD of 4.9 weeks.

The data were further analysed for two large subgroups in this study's population. Thirty-nine eyes of this series underwent 25-gauge vitrectomy for ERM stripping. Overall, there was no statistically significant difference between the mean preoperative VA $(20 / 80)$ and the mean last post-operative visit VA (20/59), regardless of final lens status $(P=0.0986)$. Mean time to baseline VA was 4.1 weeks. Further analysis was performed separating the phakic and the pseudophakic eyes. No difference in VA was seen among the 14 eyes that remained phakic throughout the study (mean preoperative VA of 20/92 and last mean post-operative visit VA of $20 / 91, P=0.99$ ), but a statistically significant difference was noted among the 25 pseudophakic eyes (mean preoperative VA of $20 / 75$ and last mean post-operative visit VA of 20/47; $P=0.0002$ ).

The second large subgroup analysis was performed on those patients who underwent a vitrectomy for the repair of a MH. There were 22 eyes in this series. The mean preoperative VA was 20/153 and the mean VA at the last post-operative visit was $20 / 46$, showing a statistically significant difference $(P<0.0001)$ independent of lens status. The mean follow-up time was 32.8 weeks, with a SD of 21.8 weeks. The mean time to baseline VA was 8.7 weeks. Of the eight eyes that remained phakic, a statistically significant difference was not seen between the mean preoperative VA (20/159) and the last mean post-operative VA obtained $(20 / 75, P=0.135)$.

Conversely, those who were pseudophakic (14) at the last follow-up had a statistically significant difference noted between the mean preoperative (20/150) and the last follow-up VA $(20 / 34 ; P<0.0001)$.

IOP measurements on POD 1 were available for 62 of 71 eyes. Forty-four of the 71 eyes $(62 \%)$ had sterile air or gas mixture injected into the eye at the end of the surgery. Overall, the mean IOP on POD 1 for all cases was $13.7 \pm 6.4 \mathrm{~mm} \mathrm{Hg}$. There was a trend for higher IOPs on POD 1 in eyes injected with sterile air and/or gas $(14.8 \pm 6.6 \mathrm{~mm} \mathrm{Hg})$ compared with those eye without intraocular injections of sterile air and/or gas $(11.7 \pm 5.6 \mathrm{~mm} \mathrm{Hg} ; P=0.07)$. On POD 1, 20 eyes $(32.3 \%)$ had an IOP less than $10 \mathrm{~mm} \mathrm{Hg}$ and nine eyes (14.5\%) had an IOP of less than $6 \mathrm{~mm} \mathrm{Hg}$. There was no significant difference in hypotony rates between surgeons for either the $10 \mathrm{~mm} \mathrm{Hg}$ cutoff $(P=0.55)$ or the $6 \mathrm{~mm} \mathrm{Hg}$ cutoff $(P=0.94)$.

The change in lens status (i.e. phakic vs pseudophakic) over the course of evaluation and treatment for all patients is demonstrated in Table 1. There were five times as many pseudophakic patients at the last post-operative visit as there were preoperatively. The development of cataracts was evaluated by a Kaplan-Meier curve of all 
Table 1 Change in lens status over the course of evaluation and treatment

\begin{tabular}{lccc}
\hline Lens stautus & $\begin{array}{c}\text { No. of patients } \\
\text { pre-operatively (\%) }\end{array}$ & $\begin{array}{c}\text { No. of patients 3 months } \\
\text { post-operatively (\%) }\end{array}$ & $\begin{array}{c}\text { No. of patients at } \\
\text { last visit (\%) }\end{array}$ \\
\hline Phakic & $63(88.8)$ & $62(87.4)$ & $29(40.9)$ \\
Pseudophakic & $8(11.2)$ & $9(12.6)$ & $42(59.1)$ \\
\hline
\end{tabular}

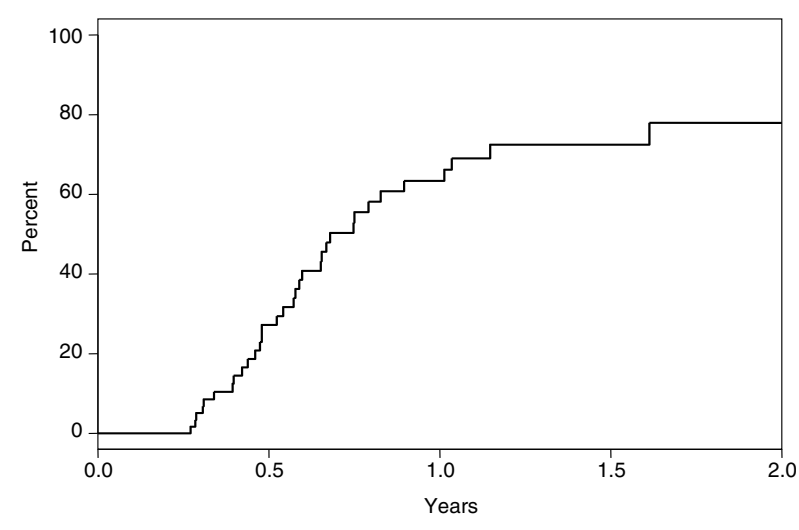

Figure 1 Kaplan-Meier curve for all cases demonstrating that $63.4 \%$ of eyes required cataract extraction at 1 year, with almost $80 \%$ requiring extraction at 2 years.

cases, which estimated that $63.4 \%$ of eyes would require cataract extraction in 1 year, and an estimated $80 \%$ by year 2 (Figure 1). Subgroup analyses with Kaplan-Meier curves, estimates the 1-year cataract extraction rate to be $67.6 \%$ for those eyes injected with gas and $54.9 \%$ for eyes without the use of gas at the conclusion of the case $(P=0.11)$ (Figure 2). The mean time to cataract surgery in this series was $30.6 \pm 18.5$ weeks (range: 11.1 weeks to 49.1 weeks after vitrectomy). A Cox proportional hazards model was used to assess the significance of age as it relates to the risk of cataract extraction and showed a hazard ratio of 1.41 for a 10-year increase in age $(P=0.03)$.

Complications reviewed included one eye (1.4\%) with an intraoperative retinal tear that was treated intraoperatively with cryotherapy. No other intraoperative or post-operative complications were noted. No cases were converted to 20-gauge vitrectomy.

\section{Discussion}

Our study demonstrates a different complication profile than earlier publications on 25-gauge vitrectomy. In our study, the common complications of hypotony and cataract did not appear to have a lasting negative impact on final VA. Mild to moderate hypotony usually resolved before any complications occurred, and cataracts could be treated with surgery successfully restoring best vision. More serious complications, including retinal detachment, retinal tear, choroidal haemorrhage and endophthalmitis were absent or rare.

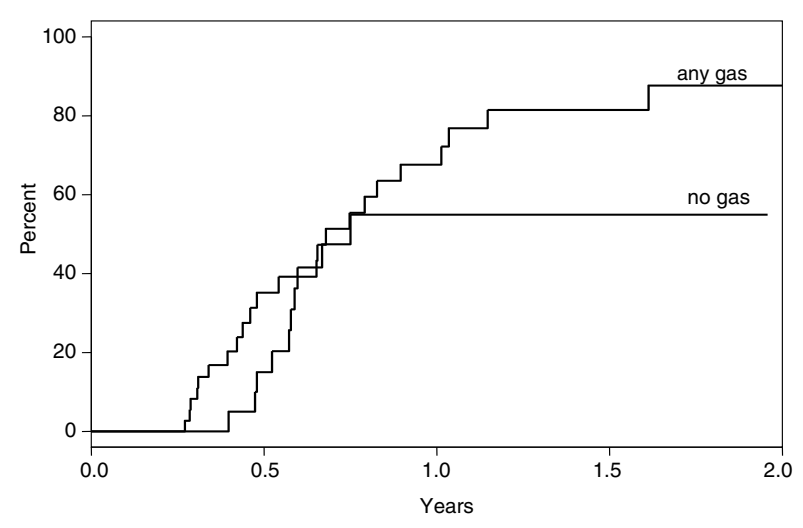

Figure 2 A Kaplan-Meier curve established that for those cases with gas left in the eye, the cataract extraction rate was $67.7 \%$ at one year. For those cases without gas in the eye, the rate was $54.9 \%$ at one year $(P=0.11)$.

We found a much higher rate of hypotony on POD 1 than most previous reports. ${ }^{1,3,4,8}$ It is unclear why this is the case, but it may be due to instrumentation, or alternatively, due to surgical technique. With regard to instrumentation, the entry system, that is, Alcon or Bausch and Lomb, would seem to be the most important factor. Each system has a different type of scleral incision. In this study, each surgeon used a different system (CAM - Alcon, RMH - Bausch and Lomb), but there was no statistically significant difference in rate of hypotony between the two surgeons, which would suggest that it was not due to the instrumentation. It could therefore be that the higher rate of hypotony is due to differences in surgical technique that the two authors share, such as a more thorough removal of the vitreous compared to others who have published previously on the topic. However, it is beyond the scope of this study to determine the exact cause of hypotony after 25-gauge vitrectomy surgery.

From the literature describing complications of hypotony after trabeculectomy, it is known that serious complications can occur, or that there is at least an increased risk of some complications, such as choroidal effusion, suprachoroidal haemorrhage, hypotony maculopathy, and possibly endophthalmitis. ${ }^{9}$ The chance of hypotony-related complications after 25-gauge surgery is poorly defined at present, and larger series may disclose more information. However, this study suggests that the risk of hypotony-related complications are not very high as none were encountered. 
Cataract development was common, and the Kaplan-Meier estimate based on the cataract surgery rate of eyes in this study suggests that by 2 years, $80 \%$ of patients will require cataract surgery. In this study, the decision to take a patient to cataract surgery was made by the evaluating physician based on the decline of vision attributed to lens changes after VA had been previously improving. Cataract formation is known to be the most frequent complication after 20-gauge vitrectomy, and rates reported vary from 12.5 to $80 \% .^{7,8,10-16}$ To date, the only other report describing a high rate of cataract formation after 25-gauge vitrectomy is by Ibarra and colleagues. ${ }^{3}$ In their study of 45 cases of 25-gauge vitrectomy, they found cataract progression to occur in $79 \%$ of phakic eyes. On the other hand, a recent publication by Rizzo and colleagues ${ }^{8}$ in which it is reported that among 26 patients undergoing 25-gauge vitrectomy, $69 \%$ of eyes had no cataract progression and none required surgery by 6 months. Their report discusses that in the 25-gauge cases a considerable amount of vitreous was left in the eye compared to their 20-gauge cases, in which they had a greater cataract progression rate. Considering Rizzo's findings and a report by Sawa and colleagues on vitrectomy-less epimacular membrane removal with complete lack of nuclear sclerosis progression, ${ }^{8,15}$ the rate of cataract formation likely depends more on the amount of vitreous removed, or possibly surgery time, than gauge of the surgical system. Thus, if a reasonably thorough vitrectomy is completed, as both surgeons in this series routinely do, there appears to be no benefit of the 25gauge systems with regard to cataract progression.

The rate of cataract formation or progression may be underestimated in series that show low rates. Our data for epimacular membrane striping show that vision, on average, did not improve significantly when all cases are considered. However, among the eyes that were pseudophakic at last VA evaluation, there was a significant improvement. Conversely, the subgroup analysis of phakic patients after both epimacular membrane stripping and $\mathrm{MH}$ surgery did not show a significant improvement in VA. Pseudophakic patients after $\mathrm{MH}$ and ERM surgery had a dramatic and highly significant VA improvement. This suggests two things: (1) the cataract surgery rate in this study likely underestimates the actual cataract formation rate, and (2) in some cases, lack of VA improvement after vitrectomy surgery may be due to lens changes and not lack of retinal or macular recovery.

Complications other than cataract were rare. There were no instances of retinal detachment, choroidal haemorrhage, or endophthalmitis, but one case of intraoperative retinal tear. These findings compare favourably to other reports. ${ }^{1,4,8}$ However, this study was not large enough to sufficiently exclude the possibility of underestimating the rate of these complications. Nevertheless, rhegmatogenous complications were not more common using the 25-gauge system as it has been suggested previously (David Williams, MD, 2005 ASRS Presentation, Montreal, Canada). It would take hundreds or thousands of prospectively followed cases to achieve sufficient statistical power to determine precise complication rates for these infrequent or rare complications.

Recovery or improvement of VA seemed accelerated when compared to the authors' experience with 20-gauge vitrectomy, though this study was not designed to answer the question of comparative time to visual recovery and no further conclusions at this time can be made. Vision recovery was faster in patients who underwent epimacular membrane stripping and not accelerated after MH surgery. Although the numbers in each subgroup may seem small for comparison, it is important to note that other papers have evaluated the 25-gauge experience with as little as 20 eyes $^{2}$ and the rate of cataract formation after pars plana vitrectomy with only 32 eyes. ${ }^{11}$ The ERM subgroup in this series was not much smaller than the total number of patients in both Rizzo's (total of 46 patients) $^{8}$ and Ibarra's studies (total of 45 patients). ${ }^{3}$

As with any study, there are limitations. This is a retrospective study without comparison group, such as 20-gauge vitrectomy cases.

In summary, cataract progression and need for cataract surgery remains a significant concern after 25-gauge vitrectomy. The rate of this complication may vary by surgery technique more so than gauge of instrumentation used. Best visual acuities were achieved after cataract extraction and intraocular lens placement. VA recovery after epimacular membrane and vitreous opacities clearing is rapid, perhaps more rapid than after 20-gauge vitrectomy; a case-control study would be helpful to prove this point. Hypotony may occur at a higher rate than reported previously, but does not appear to influence outcomes adversely.

\section{Acknowledgements}

Study performed at the Mayo Clinic, Rochester, MN, 55905, USA. Supported by research to prevent blindness, New York, NY and Mayo Foundation, Rochester, MN USA.

\section{References}

1 Fujii GY, De Juan Jr E, Humayun MS, Chang TS, Pieramici DJ, Barnes A et al. Initial experience using the transconjunctival sutureless vitrectomy system for vitreoretinal surgery. Ophthalmology (2002); 109: 1814-1820. 
2 Fujii GY, De Juan Jr E, Humayun MS, Pieramici DJ, Chang TS, Ng E et al. A new 25-gauge instrument system for transconjunctival sutureless vitrectomy surgery. Ophthalmology (2002); 109: 1807-1812; discussion 13.

3 Ibarra MS, Hermel M, Prenner JL, Hassan TS. Longer-term outcomes of transconjunctival sutureless 25-gauge vitrectomy. Am J Ophthalmol (2005); 139(5): 831-836.

4 Lakhanpal RR, Humayun MS, De Juan Jr E, Lim JI, Chong LP, Chang TS et al. Outcomes of 140 consecutive cases of 25-gauge transconjunctival surgery for posterior segment disease. Ophthalmology (2005); 112(5): 817-824.

5 Costarides AP, Alabata P, Bergstrom C. Elevated intraocular pressure following vitreoretinal surgery. Ophthalmol Clin North Am (2004); 17: 507-512.

6 de Bustros S, Thompson JT, Michels RG, Enger C, Rice TA, Glaser BM. Nuclear sclerosis after vitrectomy for idiopathic epiretinal membranes. Am J Ophthalmol (1988); 105: 160-164.

7 Panozzo G, Parolini B. Cataracts associated with posterior segment surgery. Ophthalmol Clin North Am (2004); 17: 557-568.

8 Rizzo S, Genovesi-Ebert F, Murri S, Belting C, Vento A, Cresti $\mathrm{F}$ et al 25-gauge, sutureless vitrectomy and standard 20-gauge pars plana vitrectomy in idiopathic epiretinal membrane surgery: a comparative pilot study. Graefes Arch Clin Exp Ophthalmol Jan (2006); 19: 1-8 [E-pub ahead of print].
9 Jampel HD, Musch DC, Gillespie BW, Lichter PR, Wright MM, Guire KE. Collaborative initial glaucoma treatment study group. Perioperative complications of trabeculectomy in the collaborative initial glaucoma treatment study (CIGTS). Am J Ophthalmol (2005); 140(1): 16-22.

10 Cherfan GM, Michles RG, de Bustros S, Enger C, Glaser BM. Nuclear sclerotic cataract after vitrectomy for idiopathic epiretinal membranes causing macular pucker. $A m \mathrm{~J}$ Ophthalmol (1991); 111: 434-438.

11 Hsuan JD, Brown NA, Bron AJ, Patel CK, Rosen PH. Posterior subcapsular and nuclear cataract after vitrectomy. J Cataract Refract Surg (2001); 27: 437-444.

12 Margherio RR, Cox Jr MS, Trese MT, Murphy PL, Johnson J, Minor LA. Removal of epimacular membranes. Ophthalmology (1985); 92: 1075-1083.

13 Michels RG. Vitrectomy for macular pucker. Ophthalmology (1984); 91: 1384-1388.

14 Ogura Y, Takanashi T, Ishigooka H, Ogino N. Quantitative analysis of lens changes after vitrectomy by fluorophotometry. Am J Ophthalmol (1991); 111: 179-183.

15 Sawa M, Ohji M, Kusaka S, Sakaguchi H, Gomi F, Saito Y et al Nonvitrectomizing vitreous surgery for epiretinal membrane long-term follow-up. Ophthalmology (2005); 112(8): 1402-1408.

16 Willis AW. Surgical treatment of idiopathic macular epiretinal membrane. Ophtalmologie (1989); 3: 29-30. 